\title{
DETERMINAÇÃO DOS PARÂMETROS TERMODINÂMICOS DO SISTEMA ÁGUA-ETANOL-FRUTOSE VIA DESTILAÇÃO DIFERENCIAL
}

\author{
V. R. S. ALVES ${ }^{1}$, A. BERNARDO ${ }^{1}$, L. F. MOURA ${ }^{1}$ \\ ${ }^{1}$ Universidade Federal de São Carlos, Departamento de Engenharia Química \\ vittorrodriguesantosalves@yahoo.com.br
}

\begin{abstract}
RESUMO - O presente trabalho teve dois objetivos fundamentais: buscar uma metodologia alternativa para estudar o equilíbrio líquido-vapor (ELV) e obter dados termodinâmicos precisos para a síntese do processo de produção de Frutose. Os métodos tradicionais utilizados para estudar o equilíbrio líquido-vapor, como, por exemplo, métodos estáticos ou de recirculação de fases envolvem experimentos muito detalhados e são dispendiosos. O novo método proposto baseia-se na reprodução de um processo de destilação diferencial em escala laboratorial e, a partir do balanço de massa global nesse sistema (Equação de Rayleigh), obter dados semelhantes aos que os modelos termodinâmicos sejam capazes de predizer, verificando que é possível partir de dados experimentais gerados por destilação diferencial para obter parâmetros de sistemas ainda não consolidados como é o caso da frutose em solvente hidroetanólico. Os resultados mostraram que a destilação diferencial em bancada é capaz de gerar excelentes dados do ELV e que os modelos termodinâmicos clássicos como NRLT (Non Random Two-Liquid) e Wilson, através de um ajuste de parâmetros, conseguem representar o desvio de idealidade da fase líquida para mistura ternária melhor do que os modelos preditivos.
\end{abstract}

\section{INTRODUÇÃO E MOTIVAÇÃO}

O estudo do equilíbrio líquido-vapor (ELV) é de grande importância para o desenvolvimento de processos químicos e equipamentos industriais. O ELV geralmente é estudado por uma técnica de análise chamada ebuliometria, cujos métodos podem ser estáticos ou dinâmicos (OLIVEIRA, 2003). Os métodos dinâmicos, de forma geral, necessitam que sejam agregados equipamentos para o transporte dos fluidos que circulam no sistema acarretando, assim, um custo operacional a mais (AMORIM, 2007). Já os métodos estáticos são realizados em sistema fechado sem a circulação de fases no sistema, sendo necessário um dispositivo de degaseificação o que também encarece o método (DIAS, 2016).

Paralelamente a necessidade de se estudar o ELV, esse trabalho avalia uma carência no processo desenvolvido por pesquisadores do Departamento de Engenharia Química da Universidade Federal de São Carlos (CRESTANI, 2012), onde se desenvolveu um processo de produção de frutose via uma rota de cristalização por adição de etanol como anti-solvente. A frutose possui um bom valor comercial, mas não é produzida no mercado nacional e é majoritariamente importada. 
O que impossibilitou os pesquisadores de simularem os equipamentos do processo de maneira precisa foi a ausência de um modelo termodinâmico capaz de descrever o desvio de idealidade da fase líquida de soluções hidroetanólicas contendo açúcares, principalmente a frutose, que assume um comportamento complexo em solução aquosa podendo atingir o equilíbrio químico tautomérico coexistindo sob a forma de diferentes isômeros com diferentes propriedades.

Dessa forma, buscou-se conciliar o desenvolvimento de um novo método experimental com a necessidade de estudar a termodinâmica da solução água-etanol-frutose.

\section{OBJETIVO}

Validar a método da destilação diferencial como ferramenta de estudo do ELV utilizando a solução binária água-etanol, cujos parâmetros dos modelos termodinâmicos são consistentes e bem descritos na literatura, comparando os dados experimentais com os valores previstos pelo balanço de massa do sistema e, por fim, aplicar a metodologia para estudar o equilíbrio líquido-vapor da mistura ternária composta por frutose em solução hidroetanólica.

\section{METODOLOGIA}

\subsection{Materiais e métodos}

O aparato experimental usado para realizar os ensaios de destilação diferencial é apresentado na figura 1 abaixo, composto por uma balança semi-analítica, termômetro digital, béquer com manta de aquecimento elétrico e um cronômetro.

Figura 1 - Sistema experimental de destilação diferencial

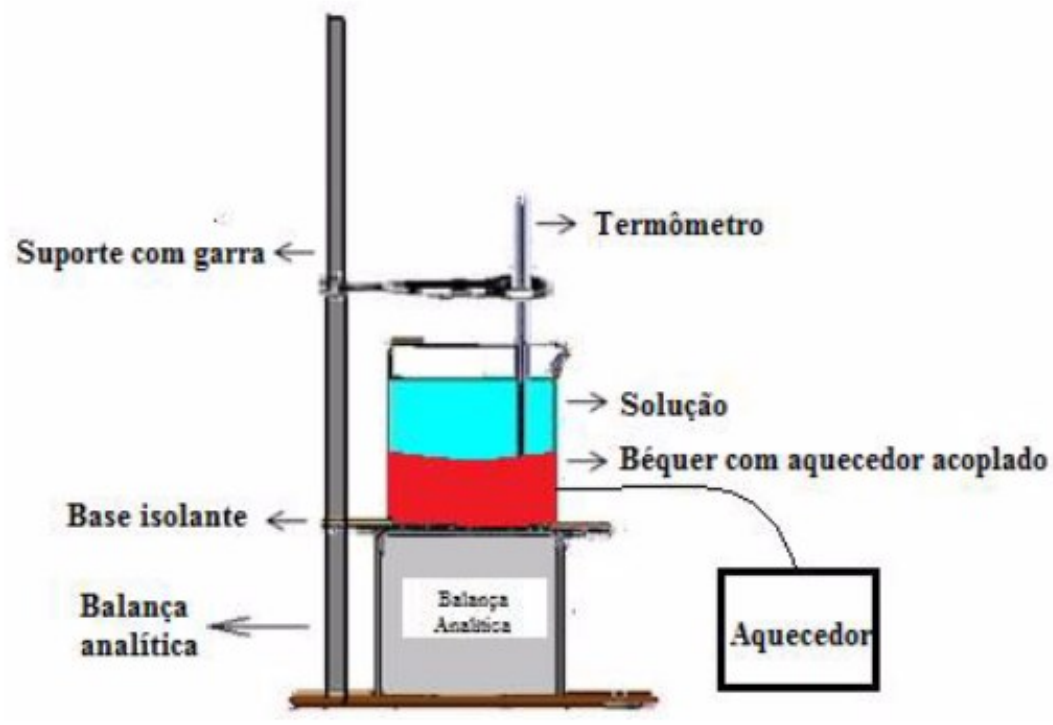

Antes de iniciar os experimentos a pressão local $(\mathrm{P})$ foi medida em manômetro. $\mathrm{O}$ sistema consistiu em monitorar apenas a fase líquida e, no momento em que a mistura entrasse em ebulição, eram retiradas amostras de aproximadamente $1 \mathrm{~mL}$ (armazenadas em 
microtubos bem vedados) utilizando uma pipeta, concomitantemente foram realizadas medidas de tempo, temperatura e massa. As medidas eram intervaladas de forma que, entre uma medição e outra, a massa do sistema reduzisse em 4\% (do seu valor inicial) para que houvesse uma variação de concentração significativa. Esse critério foi adotado a partir de observações feitas em ensaios preliminares.

Após o ensaio as amostras eram resfriadas até $21^{\circ} \mathrm{C}$ em banho termostático, pois nessa temperatura foram construídas, previamente, curvas de calibração via índice de refração $\left(\mathrm{n}_{\mathrm{D}}\right)$ para determinação da concentração tanto do binário água-etanol como do ternário águaetanol-frutose.

\subsection{Tratamento dos dados}

As amostras coletadas eram pesadas para saber qual a massa estava sendo retirada do sistema e por fim a massa experimental medida era corrigida para a massa real segundo a equação 1.

$M_{\text {real }, j}=M_{\text {medida }, j}+\sum_{k=1}^{j-1} m_{\text {amostra } a k}$

Os resultados de massa eram convertidos para base molar e por fim comparadas com o balanço global de massa do sistema conhecido como equação de Rayleigh (2).

$W_{j}=W_{0} \cdot \exp \left(\int_{x_{0}}^{x_{j}} \frac{1}{(y-x)} d x\right)$

Como a fase vapor não é monitorada, a composição $y$ é substituída pela relação de equilíbrio descrita pela lei de Raoult (3), satisfatória para as condições em que o experimento foi desenvolvido.

$$
\mathrm{x}_{\mathrm{i}} \gamma_{\mathrm{i}} \cdot P_{i}^{\text {sat }}=y_{i} \cdot P
$$

Nessa equação $\mathrm{P}_{\mathrm{i}}^{\text {sat }}$ refere-se à pressão de saturação do componente $i$ calculada usando a tradicional equação de Antoine. Assim, substituindo a composição $y_{i}$ da equação (3) na equação (2) obteve-se a equação de Rayleigh modificada (4) capaz de prever a massa $(W)$ do sistema a partir dos dados experimentais de T e composição.

$W j=W_{0} \cdot \exp \left(\int_{x_{0}}^{x_{j}} \frac{1}{x \cdot\left(\frac{\gamma_{\mathrm{i}} \cdot P_{i}^{s a t}}{P}-1\right)} d x\right)$

Dessa forma, é necessário selecionar o modelo termodinâmico apropriado para cada caso a fim de representar o coeficiente de atividade das soluções. Para água-etanol, foram utilizados os modelos de Van-Laar e Wilson, cujos parâmetros para esse binário são conhecidos e facilmente encontrados na literatura. 
Para o sistema água-etanol-frutose foram selecionados 2 modelos clássicos (NRTL e Wilson) e 3 modelos modificados que, segundo a revisão literária, mostrassem boa representação da atividade dessa mistura. Os modelos modificados foram: P\&M UNIQUAC (PRAUSNITZ, LICHTENTALER e AZEVEDO, 1999) onde uma modificação é feita na parcela combinatorial do modelo UNIQUAC e são inseridos grupos para representar as interações água-açúcar e etanol-açúcar além de algumas modificações de parâmetros como função da temperatura; O A-UNIFAC (FERREIRA, BRIGNOLE e MACEDO, 2003) que inseri uma parcela associativa no modelo UNIFAC para descrever as forças atrativas dirigidas existentes devido aos grupos $\mathrm{OH}$ em solução e o modelo mS-UNIFAC (TSAVAS, 2004) onde os açúcares em solução são enxergados em suas diversas conformações moleculares pela inserção de novos grupos funcionais.

\subsection{Determinação dos parâmetros}

A determinação dos parâmetros dos modelos NRTL e Wilson usados para a solução ternária foi feita via minimização dos quadrados resíduos entre as massas experimentais e as previstas pela equação de Rayleigh, conforme a equação (5):

$$
S Q R=\sum_{i=1}^{n}\left(W_{\text {experimental }}-W_{\text {calculado }}\right)^{2}
$$

Onde $n$ é o número de pontos totais dos experimentos e a função $S Q R$ foi resolvida via recurso Solver-Excel.

\section{RESULTADOS E DISCUSSÃO}

Os resultados comparativos para o sistema água e etanol entre os dados experimentais e os referentes modelos termodinâmicos utilizados são apresentados na figura 2 .

Figura 2 - Resultados dos ensaios para Água-Etanol
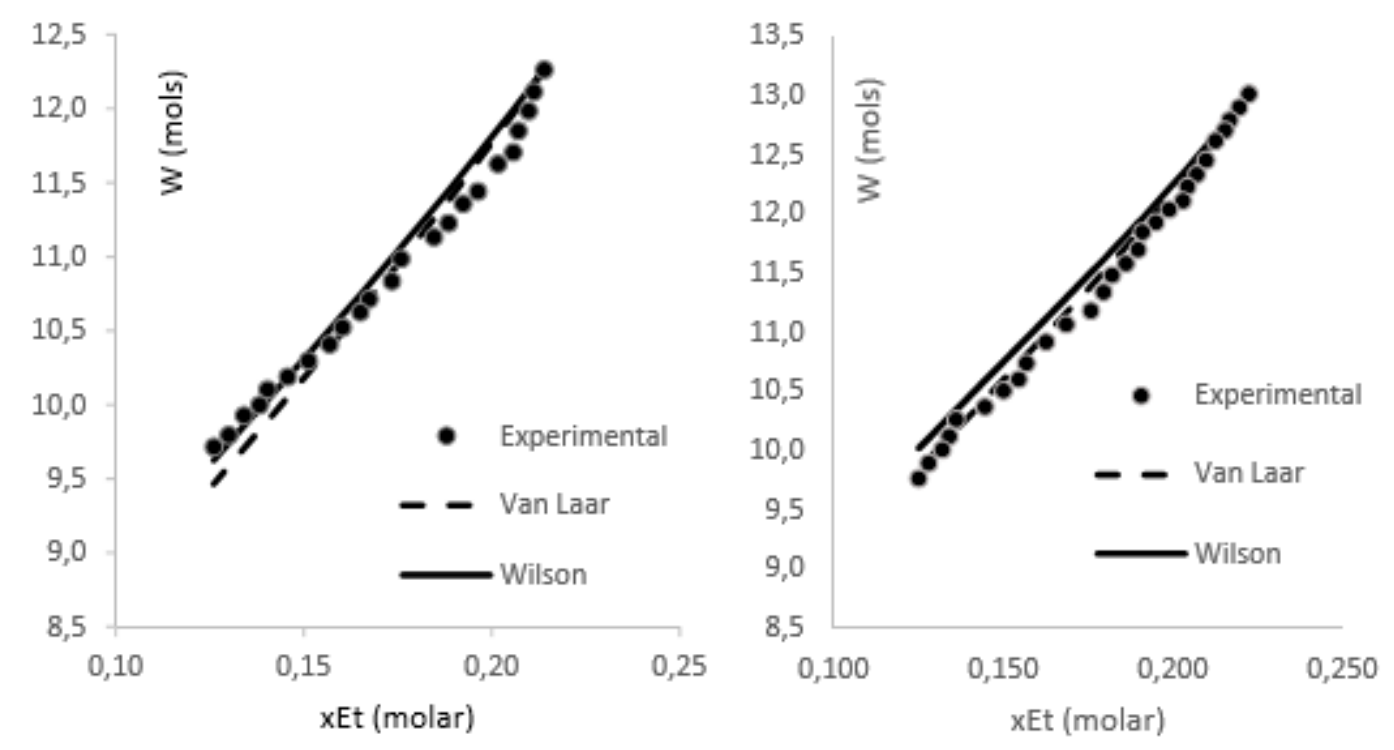
Os resultados referentes aos ensaios do sistema água-etanol-frutose são dados na figura 3 a seguir:

Figura 3 - Resultados do sistema água-etanol-frutose

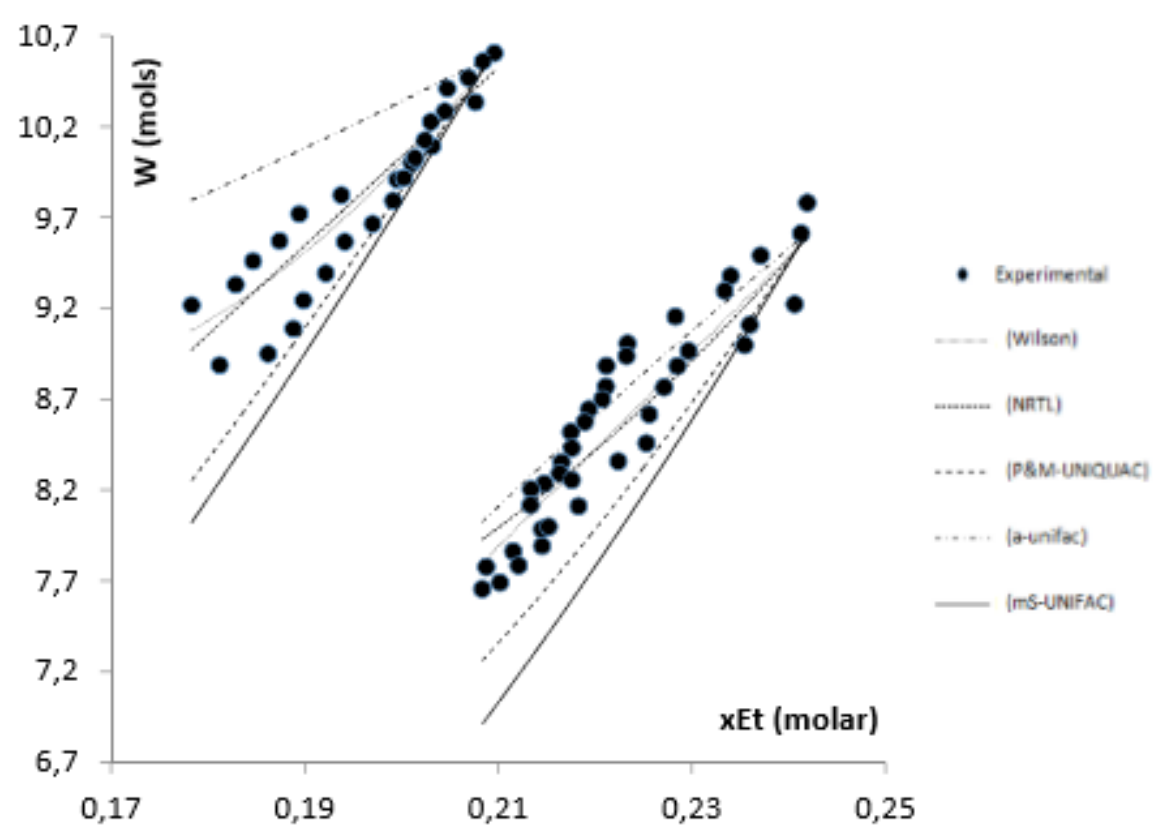

E, por fim, a tabela 1 apresenta os desvios ponderados médios e máximos entre as massas calculadas pela equação de Rayleigh (por meio dos vários modelos termodinâmicos) e a massas experimentais. Para os modelos com parâmetros ajustados esses desvios referem-se ao melhor ajuste, ou seja, na condição dos parâmetros ótimos que minimizaram a equação (5):

Tabela 1 - Desvios médios relativos das massas do sistema água-etanol-frutose.

\begin{tabular}{|c|c|c|}
\hline Modelo Termodinâmico & Desvio médio ponderado (\%) & Desvio máximo ponderado (\%) \\
\hline Wilson & 1,58 & 5,06 \\
\hline NRTL & 1,63 & 4,78 \\
\hline P\&M-UNIQUAC & 3,94 & 7,23 \\
\hline A-UNIFAC & 5,11 & 11,46 \\
\hline mS-UNIFAC & 5,83 & 10,34 \\
\hline
\end{tabular}

Pelos resultados exibido na figura 2 evidenciam-se os baixos desvios entre as massas obtidas experimentalmente e as calculadas pela equação de Rayleigh para ambos modelos termodinâmicos (Van Laar e Wilson), na prática esses desvios foram inferiores a 1\%, ou seja o método de destilação diferencial proposto se mostra eficiente na previsão do comportamento termodinâmico do sistema. Para o sistema água-etanol-frutose os melhores resultados referem-se aos modelos clássicos Wilson e NRTL que, a priori, não descrevem o comportamento de açúcares em solução, mas como a elevação do ponto de ebulição causada por açúcares em solução é desprezível à baixas concentrações (HUGOT, 1986), é possível considerar o uso desses modelos na descrição desse sistema. Assim esse resultado abre espaço 
para novas investigações a cerca do modelo termodinâmico mais preciso para esse caso, dando indícios que os modelos clássicos, principalmente o de Wilson, podem se aplicar a soluções com frutose.

\section{CONCLUSÕES DO TRABALHO}

O presente estudou possibilitou o desenvolvimento de uma nova ferramenta para estudo do ELV que é a destilação diferencial. Além disso, o estudo do sistema água-etanol-frutose, pela mesma metodologia, apontou que os parâmetros determinados dos modelos clássicos Wilson e NRTL mostram o melhor ajuste para o comportamento do sistema em equilíbrio líquido-vapor e que os modelos preditivos e modificados desenvolvidos para esse caso não foram bem representativos para dados experimentais obtidos, sendo que dentre os modelos modificados o P\&M-UNIQUAC apresentou menores desvios, mas os modelos preditivos UNIFAC apresentaram os maiores desvios tanto médio como máximo.

\section{REFERÊNCIAS}

AMORIM, J. A. Obtenção de Propriedades Físico-Químicas de Misturas de Hidrocarbonetos em Baixa e Alta Pressão Visando a Caracterização e a Modelagem. 2007. 150 f. Tese de Doutorado - Programa de Pós-graduação em Engenharia Química, Área de concentração: Engenharia de Processos em Plantas de Petróleo e Gás Natural, UFRN, Natal RN, Brasil. 2007.

CRESTANI, C. E. Integração do processo de cristalização de frutose por adição de antissolvente. São Carlos: UFSCar. 2012. 105 f. Dissertação de Mestrado, Programa de Pósgraduação em Engenharia Química, UFSCar, São Carlos-SP, Brasil, 2012

FERREIRA, O. ; BRIGNOLE, E. A. ; MACEDO, E. A. Phase Equilibria in Sugar Solutions Using the A-UNIFAC Model., Ind. Eng. Chem. Res., 42, 6212-6222, 2003.

DIAS, R. M. Estudo de equilíbrio líquido-vapor de soluções hidroetanólicas contendo frutose. São Carlos: UFSCar. 2016. 150 f. Dissertação de Mestrado, Programa de Pósgraduação em Engenharia Química, UFSCar, São Carlos-SP, Brasil, 2016

HUGOT, E. Handbook of Cane Sugar Engineering. 3 ed. Amsterdam: Elsevier, 1986. p. 494626. (Sugar Series, V.7).

OLIVEIRA, H. N. M. Determinação de Equilibrio Líquido-Vapor para sistemas Hidrocarbonetos e Desenvolvimento de uma nova Célula dinâmica. 2003. 190 f. Tese (Doutorado em Pesquisa e Desenvolvimento de Tecnologias Regionais) - Universidade Federal do Rio Grande do Norte, Natal, 2003.

PRAUSNITZ, J. M., LICHTENTHALER R. N. ; AZEVEDO E. G. Molecular Thermodynamics of Fluid- Phase Equilibria, $3^{\mathrm{a}}$ ed., Prentice Hall, Englewood Cliffs, N.J., 1999

TSAVAS et al., Phase Equilibrium Calculations in Aqueous and Nonaqueous Mixtures of Sugars and Sugar Derivatives with a Group-Contribution Model, Ind. Eng. Chem. Res., v. 43, 8391-8399, 2004. 